\title{
Studies on Specific and Hydrogen Bonding Interaction of Poly (Ethylene Glycol) with 2-Ethoxy Ethylmethacrylate Copolymer Blends
}

\author{
Manju $\mathrm{M}^{* 1}$, Veeraiah M.K ${ }^{2}$, Hemalatha $\mathrm{P}^{3}$, Prasannakumar $\mathrm{S}^{4}$ \\ ${ }^{1}$ Department of Chemistry, Sri Krishna Institute of Technology, Bangalore, India. \\ ${ }^{2,3}$ Department of Chemistry, Sri Siddhartha Institute of Technology, Tumkur, India. \\ ${ }^{4}$ Suntech Paints, Yeshwanthpur, India.
}

\begin{abstract}
The studies on the effect of polymer-polymer interactions of Poly(Ethylene Glycol) [PEG] with Methyl Methacrylate and 2-Ethoxy Ethylmethacrylate(MMA/2-EOEMA) copolymer blends showed miscibility, compatibility and specific interaction due to hydrogen bond formation between hydroxyl group from PEG and ether, ester carbonyl oxygen atom from 2-EOEMA and MMA. The studies were carried out at various blend compositions with chloroform as common solvent using viscometric, FT-IR and DSC thermal analysis methods. From viscosity data based on sign convention involved in the criterion, the values of $\mu$ and $\alpha$ for PEG with MMA/2-EOEMA copolymer blends system was computed and found to be partially positive indicating it is partially miscible and compatible for all blends composition. The compatibility and miscibility of the blends were also supported by density measurement. The properties of specific and hydrogen bonding interactions of PEG with MMA/2-EOEMA copolymer blends are studied by spectroscopic techniques such as FT-IR and the obtained results were further confirmed by DSC thermal analysis. Spectral features from above all techniques revealed that site-specific interactions are present, consistent with a significant degree of mixing of the blend components which probes mixing on a macroscopic level, which in turn imparts the miscibility to these blends.
\end{abstract}

Keywords: Blend miscibility, Compatibility, MMA/EOEMA Copolymer, PEG, Specific Interactions

\section{INTRODUCTION}

The term "polymer blend" refers to physical mixing of two or more polymers. Blending offers an attractive alternative to create new materials rather than developing totally new polymers. Many polymer producers are developing new blended products, because this strategy is usually cheaper, less time-consuming to get a product into the market and without the exorbitant cost of capital investment into a new plant $[1,2]$. The resulting blends system often exhibits properties that are superior to any one of the component polymers $[3,4]$. The basis of polymer-polymer miscibility may arise from any specific interaction such as hydrogen bonding, dipole-dipole forces and charge transfer complexes for homo-polymer mixtures. There have been various techniques of studying the polymer-polymer miscibility [5,6]. The viscometric method for the study of polymer - polymer miscibility has been suggested [7]. The variations of viscosity with blend compositions were linear for miscible blends and non-linear for immiscible blends [8, 9]. FTIR techniques are used to determine the miscibility of polymers $[10,11]$.

The acrylic monomer, methyl methacrylate $\left(\mathrm{MMA} ; \mathrm{CH}_{2}=\mathrm{C}\left(\mathrm{CH}_{3}\right) \mathrm{COOCH}_{3}\right)$ monomer has been copolymerized with various acrylate monomers to improve their properties. MMA and acrylate based copolymers are extensively studied by many authors due to their versatile use in biomedical applications as membranes for ultra filtration / drug delivery, contact lenses and anticoagulant films [12].

Another alkene monomer, 2-Ethoxyethyl methacrylate (2-EOEMA); $\mathrm{H}_{2} \mathrm{C}=\mathrm{C}\left(\mathrm{CH}_{3}\right) \mathrm{CO}_{2} \mathrm{CH}_{2} \mathrm{CH}_{2} \mathrm{OC}_{2} \mathrm{H}_{5}$ is a dual functional group monomer, which has both ether and ester groups as compared to most of the vinyl acrylic monomers; these groups not only impart flexibility into the polymer, but also improve its process ability and handling [13] and improve compatibility in blends due to hydrogen bonding [14]. Because of its soft and flexible nature, 2-EOEMA based copolymers and terpolymers could be a better candidate as surfactant [15].

MMA/EOEMA copolymers are synthesized and characterized [16]. The reactivity ratios determined by two standard methods using IR data are in good agreement with each other.

Poly (ethylene glycol) is a white, free flowing powder for creamy white flakes and is used as watersoluble lubricant for rubber moulds, textile fibers, and metal forming operations [17,18]. It is also used in water paints, paper coatings, polishes and in the ceramic industry, as well as for chromatographic stationary phases. Moreover, PEG has been considered as a promising Phase Change Materials(PCMs) for solar latent-heat thermal energy storage (LHTES) applications because of its relatively high latent heat of fusion, congruent melting and freezing behavior, suitable melting temperature range, non-corrosiveness, non-toxicity, and nondecomposition at its melting/freezing temperature range $[19,20]$. 


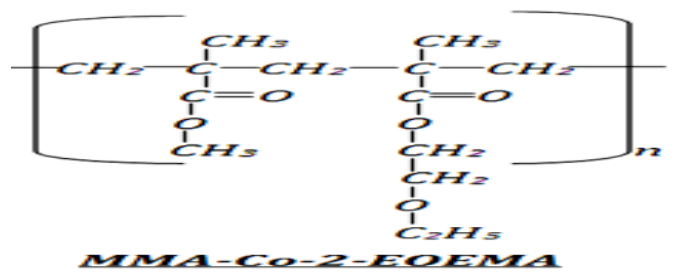

Scheme I. General Structure of MMA/EOEMA copolymer

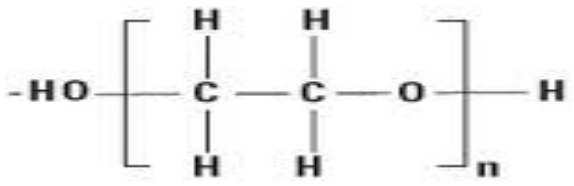

Scheme II. General Structure of PEG

The blend of PEG/PMMA has been considered as PCMs which had good thermal reliability and chemical stability. These thermal properties give the potential to PCMs for LHTES purposes such as for solar space heating and ventilating applications in buildings [21].

\subsection{Hydrogen-Bonded Polymer Blends:}

The hydrogen bond in polymer blends is an interesting and important subject of research as its presence usually enhances the miscibility of the blend. Here, the factors affecting the formation and stability of hydrogen bonds, the effects of hydrogen bonds on the miscibility, the properties of blends and methods to incorporate inter-associated hydrogen bonds into immiscible blends are reviewed, based on the work over the last two decades [22]. Among partially miscible polymer blends, hydrogen-bonded systems have played a prominent role. There exists a great structural variety of partially miscible hydrogen-bonded polymer pairs [23, 24]. In particular, a considerable amount of literature concerning the partial miscibility of PEG with other polymers has been reported [25, 26]. PEG is a proton donor that has an easily accessible hydroxyl group. It undergoes strong hydrogen-bonding interactions with proton-acceptor polymers such as poly (ethylene oxide) [27], poly (methyl methacrylate) [28], and poly (vinyl methyl ketone) [29]. In the past 10 years or so, miscibility of poly (4vinylphenol) with poly (ether) s [30] and poly (n-alkyl methacrylate)s [31] has been studied using Fourier transform infrared (FT-IR) spectroscopy.

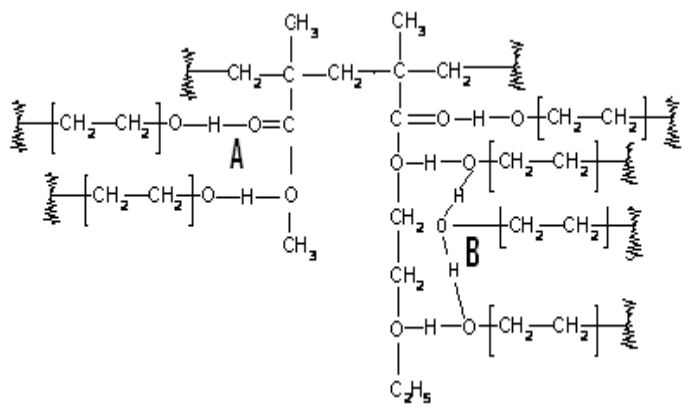

Schematic diagram showing possible types of interactions in the blends: (A) Self-association between different PEG units; (B) Interassociation between PVPh and carbonyl or ethoxy group of MMA and 2-EOEMA Copolymer.

The present investigation carried out describes the miscibility studies of MMA/EOEMA copolymer with PEG blends in chloroform by using viscosity and density at $30^{\circ} \mathrm{C}, 40^{\circ} \mathrm{C}$ and $50^{\circ} \mathrm{C}$. Solid-state compatibility of the blend was studied by FT-IR techniques. The miscibility studies of MMA/EOEMA copolymer with PEG blend might be important to enhance some of their properties. 2-EOEMA copolymer blends can be used in drug controlled release studies. The MMA/EOEMA copolymer sites on the side chain, in addition to the ester carbonyl group, there is also an ether group which is available for hydrogen bonding with -OH group of PEG blends, MMA can be copolymerized with 2-EOEMA to introduce carboxylate group that are useful for yielding a wide variety of products.

\section{MATERIALS AND METHODS}

Methyl Methacrylate, 2-Ethoxy Ethylmethacrylate, Poly Ethylene Glycol $(\mathrm{Mw}=6000)$ were obtained from Sigma Aldrich Chemicals, and Benzoyl peroxide, was obtained from S.d. Fine Chemicals. MMA and 2EOEMA both monomers were washed with dilute alkali ( $5 \% \mathrm{NaOH}$ aq. solution) followed by distilled water, dried over anhydrous $\mathrm{CaCl}_{2}$ and stored below $0^{\circ} \mathrm{C}$. Benzoyl peroxide was recrystalized from chloroform with methanol and dried in vacuum for 24hours. The other chemicals like methanol, chloroform, dimethyl formamide, acetone, 1, 4-dioxane, diethyl ether, benzene, hexane and other solvents used were AR Grade, $99 \%$ pure. They are used as received from E-Merck Chemicals, Mumbai, India. 
The free radical copolymerization of MMA with 2-EOEMA was carried out with benzoyl peroxide as an initiator and acetone as solvent. The resulting copolymer was characterized by FT-IR, $\mathrm{H}^{1}$-NMR and $\mathrm{C}^{13}$ NMR spectroscopic techniques and the formation of MMA-EOEMA copolymer is evident from spectroscopic techniques [16].

\subsection{Sample preparation and measurement}

The copolymer synthesized above [16], has been taken for further studies. A dilute polymer solution of $1 \%$ w/v was prepared for Viscometric studies. Stock solutions of homo-polymer, copolymer and the different blend compositions of MMA-EOEMA copolymer with PEG were prepared using chloroform as a common solvent. Viscosity measurements were made at $30^{\circ} \mathrm{C}$ using an Ubbelohde suspended level viscometer in the frequency $2 \mathrm{MHz}$. Density of the blend solution for different compositions of $0.5 \% \mathrm{w} / \mathrm{v}$ was measured at $30^{\circ} \mathrm{C}$, $40^{\circ} \mathrm{C}$ and $50^{\circ} \mathrm{C}$,using pycnometer for dilute solutions and specific gravity bottle for concentrated solutions. The copolymer blend solutions in different concentrations were poured into a Petri dish kept on a flat tiled table that was leveled by spirit leveler. It was allowed to dry overnight at room temperature. The films were then peeled off, covered with aluminum foil and kept in the desiccators till further analysis. These films are of the order of microns and transparent. The samples dried by an infrared lamp were made to fine powder, mixed with $\mathrm{KBr}$, pressed to give a pellet and used for the FT-IR analysis. FT-IR studies were made with SHIMADZU spectrophotometer.

\section{RESULTS AND DISCUSSION}

\section{III.1 Viscometric studies}

The reduced viscosities of copolymer and their blends with various compositions were found out at $30^{\circ} \mathrm{C}$ are shown in Table 1. The reduced viscosities of the pure polymers and their blend compositions were plotted against concentrations, as shown in Fig-1. It has been observed that MMA-EOEMA copolymer /PEG blends are partially miscible over some composition range (0.1 to 0.6$)$ were linear, no cross-over is seen showing that blends are compatible and in some composition range ( 0.6 to 1.0) were non-linear and hence noncompatible.

Table -1 Reduced viscosity data for copolymer/PEG and their blends in chloroform at $30^{\circ} \mathrm{C}$

\begin{tabular}{|l|l|l|l|l|l|l|l|l|l|l|}
\hline $\begin{array}{l}\text { Con } \\
\mathrm{c} \\
(\mathrm{C}) . \\
\mathrm{g} \\
/ 100 \\
\mathrm{Cm}^{3}\end{array}$ & PEG & $\begin{array}{l}\text { copoly } \\
\text { /PEG }\end{array}$ & $\begin{array}{l}\text { Copol } \\
\text { y/PEG }\end{array}$ & $\begin{array}{l}\text { Copol } \\
\text { y/PEG }\end{array}$ & $\begin{array}{l}\text { Copol } \\
\text { y/PEG }\end{array}$ & $\begin{array}{l}\text { Copol } \\
\text { y/PEG }\end{array}$ & $\begin{array}{l}\text { Copol } \\
\text { y/PEG }\end{array}$ & $\begin{array}{l}\text { Copol } \\
\text { y/PEG }\end{array}$ & $\begin{array}{l}\text { Copol } \\
\text { y/PEG }\end{array}$ & $\begin{array}{l}\text { Copol } \\
\text { ymer }\end{array}$ \\
\hline 0.1 & 0.3394 & 0.3594 & 0.3781 & 0.4042 & 0.4261 & 0.4482 & 0.4692 & 0.4982 & 0.5417 & 0.5615 \\
\hline 0.2 & 0.3647 & 0.3824 & 0.4081 & 0.4365 & 0.4578 & 0.4768 & 0.5089 & 0.5349 & 0.5789 & 0.5990 \\
\hline 0.3 & 0.3904 & 0.4046 & 0.4423 & 0.4678 & 0.4883 & 0.5093 & 0.5453 & 0.5753 & 0.6173 & 0.6341 \\
\hline 0.4 & 0.4195 & 0.4395 & 0.4643 & 0.4901 & 0.5198 & 0.5412 & 0.5806 & 0.6123 & 0.6539 & 0.6715 \\
\hline 0.5 & 0.4466 & 0.4696 & 0.4898 & 0.5175 & 0.5451 & 0.5751 & 0.6151 & 0.6471 & 0.6912 & 0.7088 \\
\hline 0.6 & 0.4737 & 0.4938 & 0.5102 & 0.5467 & 0.5765 & 0.6095 & 0.6475 & 0.6848 & 0.7248 & 0.7439 \\
\hline 0.7 & 0.4978 & 0.5199 & 0.5348 & 0.5684 & 0.6091 & 0.6414 & 0.6824 & 0.7196 & 0.7596 & 0.7770 \\
\hline 0.8 & 0.5212 & 0.5412 & 0.5536 & 0.5981 & 0.6298 & 0.6778 & 0.7169 & 0.7569 & 0.7969 & 0.8165 \\
\hline 0.9 & 0.5454 & 0.5645 & 0.5761 & 0.6173 & 0.6587 & 0.7096 & 0.7497 & 0.7969 & 0.8289 & 0.8495 \\
\hline 1 & 0.5702 & 0.5912 & 0.6021 & 0.6411 & 0.6903 & 0.7393 & 0.7893 & 0.8363 & 0.8663 & 0.8868 \\
\hline
\end{tabular}




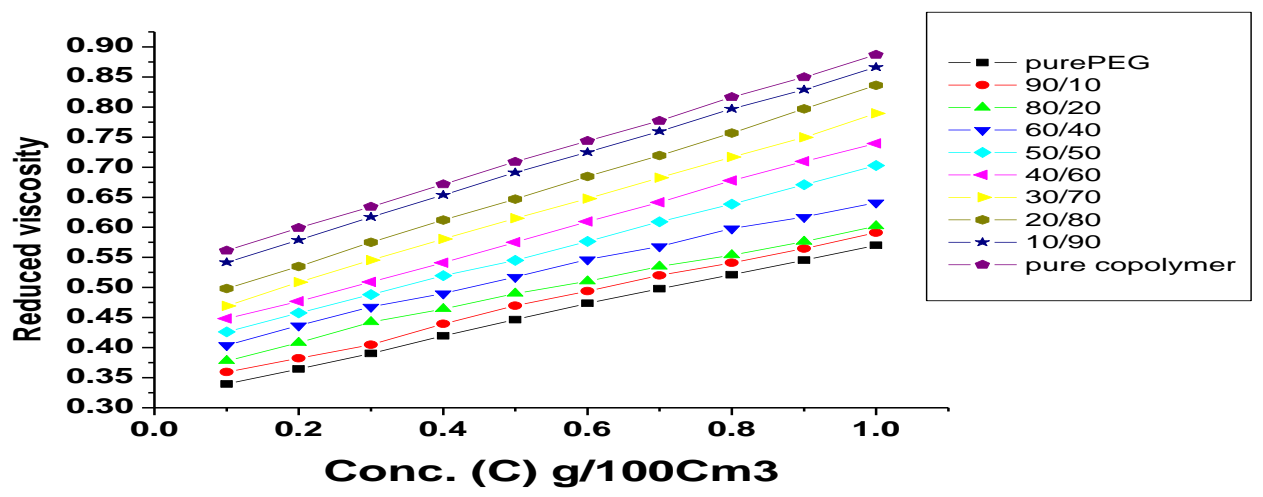

Figure-1 Reduced viscosity verses concentration of copolymer/PEG blends

Table-2 Viscometric data i.e. [ $\boldsymbol{\eta}], \mathbf{b}, \mathbf{K H}, \boldsymbol{\mu}$ and $\boldsymbol{\alpha}$ of copolymer/PEG blends

\begin{tabular}{|c|c|c|c|c|c|c|c|c|c|c|}
\hline $\begin{array}{c}\% \text { of } \\
\text { the } \\
\text { Blends }\end{array}$ & Slope & $\begin{array}{c}\text { Wt. of } \\
\text { Copolymer }\end{array}$ & $\begin{array}{c}\text { Wt. } \\
\text { of } \\
\text { PEG }\end{array}$ & b & $\overline{\mathbf{b}}$ & 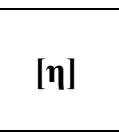 & $\Delta \mathbf{B}$ & KH & $\mu$ & $\alpha$ \\
\hline $\begin{array}{l}\text { co } \\
\text { Poly }\end{array}$ & 0.7234 & 1 & 0 & 0.7234 & 0.7234 & 0.6349 & 0 & 0 & 0 & 0 \\
\hline $\mathrm{A}$ & 0.72 & 0.9 & 0.1 & 0.7207 & 0.7025 & 0.624 & 0.1011 & 1.8509 & 2.9959 & 1.8374 \\
\hline $\mathrm{B}$ & 0.7356 & 0.8 & 0.2 & 0.719 & 0.6817 & 0.6078 & 0.1166 & 1.9463 & 3.4553 & 1.8888 \\
\hline $\mathrm{C}$ & 0.7059 & 0.7 & 0.3 & 0.6973 & 0.6608 & 0.5818 & 0.0869 & 2.06 & 2.5751 & 1.89795 \\
\hline $\mathrm{D}$ & 0.6377 & 0.6 & 0.4 & 0.6489 & 0.6399 & 0.5535 & 0.0188 & 2.118 & 0.5571 & 1.8567 \\
\hline E & 0.575 & 0.5 & 0.5 & 0.597 & 0.6191 & 0.5249 & $\begin{array}{c}- \\
0.0442\end{array}$ & 2.1181 & 3098 & 1.6818 \\
\hline $\mathrm{F}$ & 0.5405 & 0.4 & 0.6 & 0.5605 & 0.5982 & 0.4954 & $\begin{array}{c}- \\
0.0785 \\
\end{array}$ & 2.2838 & $\begin{array}{c}- \\
2.3262\end{array}$ & -0.6107 \\
\hline $\mathrm{H}$ & 0.4717 & 0.2 & 0.8 & 0.5093 & 0.5564 & 0.4713 & $\begin{array}{c}- \\
0.1472 \\
\end{array}$ & 2.2929 & -4.362 & -0.9093 \\
\hline I & 0.5227 & 0.1 & 0.9 & 0.5182 & 0.5356 & 0.4645 & $\begin{array}{c}- \\
0.0967\end{array}$ & 2.4017 & $\begin{array}{c}- \\
2.8656\end{array}$ & -1.7889 \\
\hline PEG & 0.5147 & 0 & 1 & 0.5147 & 0.5147 & 0.4512 & 0 & 2.5283 & 0 & 0 \\
\hline
\end{tabular}

MMA/EOEMA copolymer and PEG blends were found to be miscible only when MMA/EOEMA copolymer content is $60 \%$ and above, below this critical concentration, due to the phase separation, smaller slope value was observed in the Huggin's plot. In order to quantify the miscibility of polymer blends, [5] suggested the general expression for interaction parameter when the polymers are mixed in weight fractions $\mathrm{w}_{1}$ and $\mathrm{w}_{2}$ as

$$
\Delta B=\frac{b-\bar{b}}{2 w_{1} w_{2}}
$$

Where $\bar{b}=\mathrm{w}_{1} \mathrm{~b}_{11}+\mathrm{w}_{2} \mathrm{~b}_{22}$ where $\mathrm{b}_{11}$ and $\mathrm{b}_{22}$ are the slopes of the viscosity curves for the Components and $b$ is related to Huggin's coefficient $\mathrm{KH}$ as,

$$
\mathbf{b}=\mathbf{K H}[\boldsymbol{\eta}]^{2}
$$

Where $[\eta]$ is the intrinsic viscosities of blend solution, for ternary system, it is also given by

$$
b=w_{1}{ }^{2} b_{11}+w_{2}{ }^{2} b_{22}+2 w_{1} w_{2} b_{12}
$$

Where $b_{12}$ is the slope for blend solution. However, Chee's theory fails to account for the experimental data when intrinsic viscosities of pure components are far apart. In such cases he defined a more efficient parameter $(\mu)$ to predict compatibility, 


$$
\mu=\frac{\Delta B}{\left\{[\eta]_{2}-[\eta]_{1}\right\}^{2}}
$$

Where $\mu$ is Chee's interaction parameter, $[\eta]_{1}$ and $[\eta]_{2}$ are intrinsic viscosities of pure component solutions. The polymer blend is miscible if $\mu \geq 0$ and immiscible when $\mu<0$. Later [6] have suggested a most satisfactory new equation for the determination of polymer miscibility as

$$
\alpha=K_{m}-\frac{K_{1}[\eta]_{1}^{2} W_{1}^{2}+K_{2}[\eta]_{2}^{2} W_{2}^{2}+2 \sqrt{K_{1} K_{2}}[\eta]_{1}[\eta]_{2} W_{1} W_{2}}{\left.\{\eta]_{1} W_{1}+[\eta]_{2} W_{2}\right\}^{2}}
$$

Where $\alpha$ is Sun's interaction parameter; $\mathrm{K} 1, \mathrm{~K} 2$ and $\mathrm{Km}$ are the Huggins constants for individual components 1, 2 and blend respectively. The long-range hydrodynamic interactions are considered while deriving the equation. The polymer blend is miscible if $\alpha \geq 0$ and immiscible when $\alpha<0$.

In the present study the value of $\mu$ and $\alpha$ for MMA-EOEMA copolymer and PEG computed to be positive for few blends composition, predicting the partial miscibility and compatibility as show in the Table.2

\section{III.2 Density studies:}

\begin{tabular}{|c|c|c|c|}
\hline \multirow{2}{*}{$\begin{array}{c}\text { Composition } \\
\text { of } \\
\text { copolymer/PEG }\end{array}$} & \multicolumn{3}{|c|}{$\begin{array}{l}\text { Density of the Blend solutions at } \\
\text { different temperature }\end{array}$} \\
\hline & $30^{0} \mathrm{C}$ & $40^{\circ} \mathrm{C}$ & $50^{\circ} \mathrm{C}$ \\
\hline copolymer & 1.44771 & 1.44804 & 1.448418 \\
\hline $\mathrm{A}$ & 1.44754 & 1.447856 & 1.448231 \\
\hline B & 1.44738 & 1.447688 & 1.448031 \\
\hline $\mathrm{C}$ & 1.44719 & 1.4475 & 1.447843 \\
\hline $\mathrm{D}$ & 1.447028 & 1.447319 & 1.447649 \\
\hline $\mathrm{E}$ & 1.44686 & 1.447144 & 1.447468 \\
\hline$F$ & 1.446691 & 1.44697 & 1.44728 \\
\hline G & 1.446905 & 1.447157 & 1.447481 \\
\hline $\mathrm{H}$ & 1.447119 & 1.447416 & 1.447675 \\
\hline $\mathrm{I}$ & 1.447235 & 1.447468 & 1.447765 \\
\hline PEG & 1.447267 & 1.447558 & 1.447856 \\
\hline
\end{tabular}

Table-3 Density data for copolymer/PEG and their blends in chloroform at $30^{\circ} \mathrm{C}, 40^{\circ} \mathrm{C} \& 50^{\circ} \mathrm{C}$

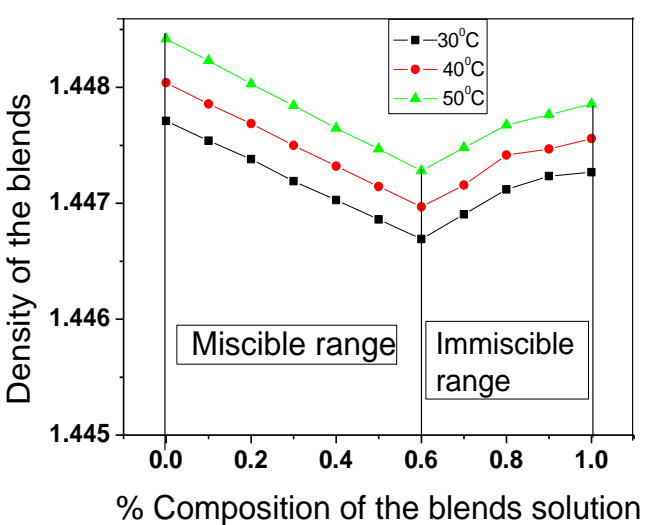

Figure-2 Density verses \% composition of the blends

The blends of MMA/EOEMA copolymer /PEG are partially miscible over range of composition because of strong intermolecular interaction based on strong hydrogen bonding. They are shown in Table-3 and Fig-2. Thus, simple density measurement studies give information regarding the partial miscibility of the blends.

\section{III.3 FT-IR Spectroscopy:}

FT-IR spectroscopy has been used to identify the specific intermolecular interactions occurred in MMA/EOEMA copolymer / PEG blends. FT-IR analysis confirmed that strong intermolecular hydrogen bonding interactions between the ether groups of the copolymer and the hydroxyl groups of the PEG. Schematic representation of the potential favorable intermolecular hydrogen bonding interaction occurred in copolymer / PEG blends is shown in the Fig-3

FT-IR investigation was performed on MMA/EOEMA copolymer, PEG and their blends with different composition ratio and the spectrums are compared. It is found that stretching vibration bands of both $\mathrm{O}-\mathrm{H}$ groups in pure $\mathrm{PEG}$ at $3359 \mathrm{~cm}^{-1}$ and $\mathrm{C}=\mathrm{O}$ group in pure MMA/EOEMA copolymer at $1731.70 \mathrm{~cm}^{-1}$, shift observable in the MMA/EOEMA copolymer /PEG blends to the direction of higher wave number at 3464.39 $\mathrm{cm}^{-1}-3502.31 \mathrm{~cm}^{-1}$ and $1731.25 \mathrm{~cm}^{-1}$ to $1731.74 \mathrm{~cm}^{-1}$ respectively. The shift of carbonyl stretching band may result from intermolecular hydrogen bonding and contribute to the enhancement of the state of miscibility of blends.Table-4 The FTIR spectroscopy demonstrated that there is strong intermolecular hydrogen bonding between ether group of MMA/EOEMA copolymer and free hydroxyl group of PEG.The FTIR spectra the peak 
at $1110.75 \mathrm{Cm}^{-1}-1125.44 \mathrm{Cm}^{-1}$ is due to the strong absorption caused by asymmetric C-O-C stretching in vinyl ether of 2-EOEMA unit. The ether group of MMA-EOEMA copolymer is hydrogen bonded with hydroxyl group of PEG blends with various blend composition (A, B, C, D, E, F, G, H, I) was recorded at room temperature. In the present study, we focused only on hydrogen bonding.

Table-4 Hydrogen bonding interaction data for MMA-EOEMA copolymer/PEG and their blends

\begin{tabular}{|c|l|l|}
\hline $\begin{array}{l}\text { Blend } \\
\text { composition } \\
\text { of copolymer } \\
\text { /PEG }\end{array}$ & $\begin{array}{l}\text { Hydrogen } \\
\text { bonded } \\
\text { ether group }\end{array}$ & $\begin{array}{l}\text { Hydrogen } \\
\text { bonded } \\
\text { Hydroxyl } \\
\text { group }\end{array}$ \\
\hline copolymer & 1125.44 & \\
\hline A & 1122.76 & 3502.31 \\
\hline B & 1119.46 & 3502.04 \\
\hline C & 1120.49 & 3501.85 \\
\hline D & 1118.12 & 3501.79 \\
\hline E & 1117.47 & 3501.58 \\
\hline F & 1115.13 & 3492.06 \\
\hline G & 1113.54 & 3482.20 \\
\hline H & 1111.77 & 3474.90 \\
\hline I & 1110.75 & 3464.39 \\
\hline
\end{tabular}

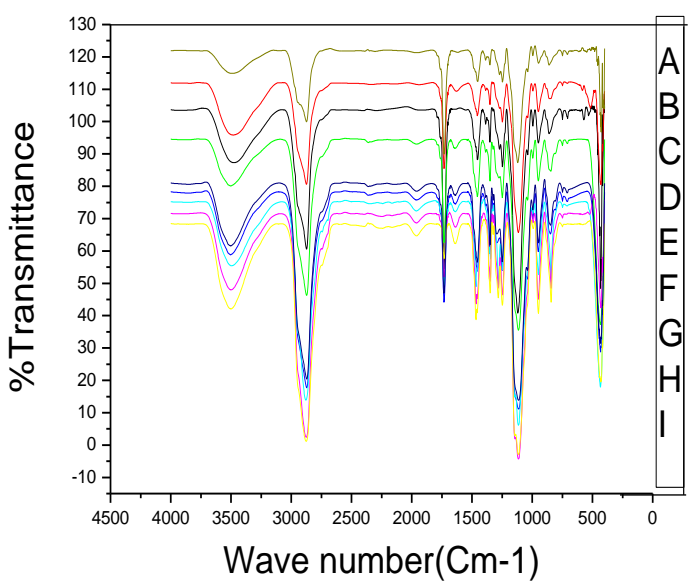

Figure-3: FTIR spectra of hydrogen bonded ether and hydroxyl group of copolymer/PEG blends with various composition

This figure qualitatively demonstrates that the amount of intermolecular hydrogen bonded ether groups of the MMA-EOEMA copolymer are increased as the PEG composition of the PEG blend increases. Hence, it shows hydrogen-bonding effect on blend compatibility and miscibility

\section{Thermal Analysis:}

Thermal analysis were performed on a Universal 4.7A TA Instrument DSC Q200V24.9Buld121 with a thermal analyst 2000 at a heating rate of $20^{\circ} \mathrm{C} / \mathrm{min}$. to destroy any thermal history and subsequently quenched into liquid nitrogen. The glass transition temperature was taken as the midpoint of the heat flow change during second heating. The weight of the sample used was typically 5-10 mg. A common method to assess the miscibility of polymer blends is to measure the glass transition temperature of the blend component which represents the onset of the cooperative segmental motion of the component copolymers with PEG blends, are shown in Table-5 and Figure-4.

Table-5 Determination of Tg by DSC thermal analysis of copolymer/PEG blends

\begin{tabular}{|c|c|c|}
\hline $\begin{array}{c}\text { Wt Fraction } \\
\text { of } \mathrm{A}\end{array}$ & $\begin{array}{c}\text { Wt Fraction } \\
\text { of } \mathrm{B}\end{array}$ & $\begin{array}{c}\text { Resultant Tg } \\
\left({ }^{0} \mathrm{C}\right)\end{array}$ \\
\hline 0 & 1 & 33.3 \\
\hline 0.1 & 0.9 & 26.6 \\
\hline 0.2 & 0.8 & 20.2 \\
\hline 0.3 & 0.7 & 14.0 \\
\hline 0.4 & 0.6 & 8.1 \\
\hline 0.5 & 0.5 & 2.4 \\
\hline 0.6 & 0.4 & -2.9 \\
\hline 0.7 & 0.3 & -8.2 \\
\hline 0.8 & 0.2 & -13.2 \\
\hline 0.9 & 0.1 & -18.1 \\
\hline 1 & 0 & -22.7 \\
\hline
\end{tabular}

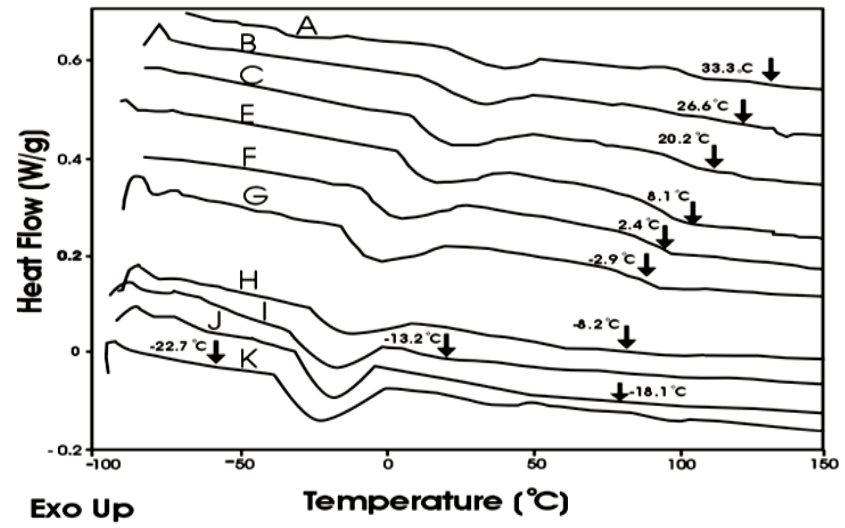

Figure-4. DSC thermograms of copolymer with PEG blends 


\section{CONCLUSIONS}

It has been observed that MMA-EOEMA copolymer/PEG blends are partially miscible over some composition range. They were linear, no cross-over is seen showing that blends are compatible and in some composition range were non-linear and hence non-compatible.

The values calculated from the viscometric data for $\boldsymbol{\mu}$ and $\boldsymbol{\alpha}$ parameter. The $\mu$ and $\alpha \geq 0$ polymer blend is miscible and $\mu$ and $\alpha<0$ polymer blend is immiscible, hence suggests partially Compatible and miscibility for MMA-EOEMA copolymer / PEG blends. The compatibility and miscibility of blends were also supported by density measurement. FTIR spectroscopy also revealed specific interaction between ester carbonyl and ether group of MMA-EOEMA copolymer and Hydroxyl group of PEG blend. It is due to Hydrogen bonding effect on blends which shows partial computability and miscibility over all percentage of compositions; above all concepts are further confirmed by DSC thermal analysis. The partially miscible and compatible copolymers with PEG blends are extensively used in biomedical applications.

\section{REFERENCES}

[1] Long Yu., Katherine Dean., Lin Li. 2006. Prog. Polym. Sci. 31, 576-602.

[2] Ramesh S., CW Liew., E Morris. 2010. Thermochimica Acta. Elsevier, 511, 1-2, 140-146.

[3] Mruthyunjaya Swamy T. M., Siddaramaiah. 2007. J. Applied Polymer Sci. 104, 3, 2048-2053.

[4] Ramesh S., A.H. Yahaya., A.K. Arof. 2002. Solid State Ionics 148, 483- 486.

[5] Chee K. K. 1990. European Polymer Journal, 26, 423-426.

[6] Sun, Z., Wang W., \& Feng Z. 1992. European Polymer Journal, 51, 1259.

[7] Paladhi R. \& Singh R. P. (1994a). European Polymer Journal, 51, 1559.

[8] Paladhi R. \& Singh R. P. (1994b). Journalof Applied Polymer Science, 30, 25, 251.

[9] Varada Rajulu V \& Mabusab P. 1996. European Polymer Journal, 32, 267-268.

[10] Changhua Liu \& Chaobo Xiao. 2005. Journal of Applied Polymer Science, 95, 1405-1411.

[11] Manisara Peesan \& Pitt Supaphol. 2005. Carbohydrate Polymers. 60, 343-350.

[12] Sheng Q.N., Fen R., Chao H., Peng F. Z., Xiao H.W., Jie, L., Chang S.Z. 2011, Chinese chem.Letters, 22(3), 370-373.

[13] Vijay K.S., Prasanna Kumar S., MusturappaT.E., Mahadevan K.M., Sherigara B. S. 2007. J. of MacroMolecu Lar Science, part A: Pure and Appl. Chemistry, 44, 1161-1169.

[14] SenihaG.F., YusufY., Tuncer Erciyes A. 2006, Prog. Polym. Sci., 31, 633-670.

[15] Sanmathi C.S., Prasannakumer S., Sherigara B.S. 2004, Bull. Mater. Sci., 27, (3), $243-249$.

[16] Manju M., Veeraiah M.K., Prasannakumar S., Madegowda N.M and Sherigara B.S. American Journal of Polymer Science.2012, 2(3), 22-27.

[17] Qi Song Shi., Jian Xiang Yu., Tai Qi Liu. 2011. Advanced Materials Research, 183-185, 2082-2085

[18] Cemil Alkan., Ahmet Sari., Orhan Uzun. 2006. AIChE Journal. 52, 9, 3310-3314.

[19] Pielichowski K., Flejtuch K. 2003. Macromolecular Material Eng. 288:259-264.

[20] Xiao M., Feng B., Gong K. 2002. Energy Conversion Management. 43:103-108.

[21] Ahmet Sari., Cemil Alkan., Ali Karaipekli., Orhan Uzun. 2010. J. of Applied Poly. Sci. 116, 2, 929 933.

[22] Nisa V. Salim., Tracey Hanley., Qipeng Guo. 2010. Macromolecules. 43, 7695-7704.

[23] Mazurek, M.; Leir C.M.; Journal of Applied Polymer Science, 2010, 117(2), 982-995.

[24] Chen L. L., Wan C. C., ChunS. L., Yi C. S., Chih F. H., Shiao W. K., Feng C. C.2005, Macromolecules, 38, 6435-6444.

[25] Hexig A., Bo Z., Yoshio I. 2007, Journal of Applied Polymer Science, 106(3), 2025-2030.

[26] Wen-Ping Hsu. 2006, Journal of Applied Polymer Science, 101(10, 643-652.

[27] Derek I.H., Boualem H., Steven R. K. 2003, J Polym Sci Part B: Polym Phys., 41: 135-138.

[28] Gui X.H., Xiao A.C., Xiao M.S. 2011, Advanced Materials Research, 337, 184-187.

[29] Chuan Q., Alfredo T. N. P., Laurence A. B, 1991, Macromolecules, 24 (3), 666-670.

[30] Serman C., Xu Y., Painter P. C., Coleman M. M. 1991, Polymer, 32, 516.

[31] Coleman M. M., Serman C., Painter P. C. 1991, Polymer, 32, 1049. 